\title{
Identification of transcriptional targets of healthspan in adipose tissue and modulation by resveratrol
}

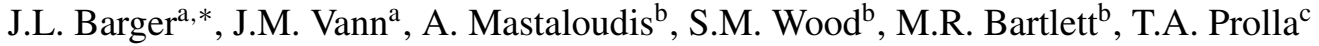 \\ and R. Weindruch ${ }^{\mathrm{d}}$ \\ ${ }^{a}$ LifeGen Technologies LLC, Madison, WI, USA \\ ${ }^{\mathrm{b}}$ Nu Skin Enterprises, Provo, UT, USA \\ ${ }^{c}$ Departments of Genetics and Medical Genetics, University of Wisconsin, Madison, WI, USA \\ ${ }^{\mathrm{d}}$ Department of Medicine, University of Wisconsin and William S. Middleton Memorial Veterans Hospital, \\ Madison, WI, USA
}

\begin{abstract}
Although calorie restriction (CR) slows the aging process in diverse species, CR is not a pragmatic strategy to attenuate aging in free-living humans. Accordingly, there is growing interest in identifying CR mimetics-compounds that elicit the salutary effects of CR without a reduction in energy intake. Because increased white adipose tissue (WAT) is associated with accelerated onset of many age-associated diseases, and because a reduction of WAT is a consistent feature of CR, diet-induced changes in WAT bioactivity may be attractive targets for assessing the efficacy of CR mimetics. We analyzed gene expression datasets from WAT of obese mice and humans and found a significant modulation of 22 pathways indicating decreased mitochondrial function and an up-regulation of the immune response and lysosomal structure. The change with obesity was abrogated by long-term $\mathrm{CR}$ in WAT of both mice and rats for 13 of the 22 pathways. For those 13 pathways, consumption of the putative CR mimetic resveratrol both opposed the effect obesity and mimicked the effect of CR for 11 and 10 pathways in human and mouse adipose tissue, respectively. Numerous studies have shown that resveratrol delays diverse aspects of the aging process, suggesting that our observed transcriptional response in WAT is indicative of increased healthspan. We propose that these pathways may be used as a framework to screen for novel CR mimetics.
\end{abstract}

Keywords: Calorie restriction, obesity, mitochondria, immune response, nutrigenomics, diabetes

\section{Introduction}

The ability of a calorie restricted (CR) diet to extend lifespan was first demonstrated in rats in 1935 [1]; since that time, CR has been shown to increase lifespan and slow the aging process in species ranging from yeast, flies, worms and mice (reviewed in 2). While the two studies of long-term $\mathrm{CR}$ in nonhuman primates are equivocal with regards to extending maximum lifespan [3, 4] both studies (as well as numerous studies in other mammals) have repeatedly demonstrated that CR slows the aging process as indicated by a decreased

${ }^{*}$ Corresponding author: J.L. Barger, LifeGen Technologies LLC, Madison, WI 53719, USA. E-mail: Jamie.L.Barger@gmail.com. risk and/or delayed onset of age-associated diseases, including cardiovascular disease [5], sarcopenia [6], type 2 diabetes [7], neurodegeneration and some cancers [3].

White adipose tissue (WAT) is increasingly being recognized as an active endocrine organ that secretes numerous cytokines that circulate in the blood and regulate peripheral physiology [8]. Interestingly, changes in WAT bioactivity observed with obesity have been implicated in the pathogenesis of many of the same age-associated diseases that are attenuated by CR [9-13]. Taken together, these observations suggest a mechanistic link between the activity of WAT and risk of disease [14]. Although CR-induced changes in WAT function may play a role in slowing the aging process, 
long-term dietary restriction does not represent a realistic therapeutic strategy in humans. As a result, there is a growing interest in identifying CR mimetics, defined as those compounds that induce the beneficial effects of a CR diet without requiring a dramatic reduction in energy intake $[15,16]$. The phytoalexin resveratrol, found in grape skins, red wine and certain plants, has received much attention in this field largely due to the early observations that moderate wine consumption appears to be protective against cardiovascular disease [17] and that resveratrol inhibited carcinogenesis [18].

More recent studies have shown that resveratrol consumption extends lifespan of yeast [19], flies, worms [20], fish [21] and mice on a high fat diet [22]. Although mice on a standard diet containing resveratrol did not live longer than controls [23, 24], resveratrol-treated mice have an improved healthspan; in addition, global patterns of gene expression strongly resembled CR mice in multiple tissues $[22,23,25,26]$. Several clinical trials of resveratrol consumption have been reported in the literature, however gene expression datasets from these studies have not been analyzed using a single, consistent methodology. Thus there is a need for a comprehensive meta-analysis of WAT gene expression using a single statistical approach. The current study sought to fill this gap by asking [1] is there is a common pattern of gene expression in WAT that is sensitive to, and inversely regulated by, obesity and CR; and [2] does resveratrol consumption recapitulate the effect of $\mathrm{CR}$ in WAT of mice and humans?

\section{Methods}

\subsection{Animals}

Male C57BL/6 x C3H/He F1 hybrid mice were purchased at six weeks of age (Harlan Laboratories), individually housed in a specific pathogen free facility and fed a diet based on the AIN-93M formulation (Bio-Serv, Frenchtown, NJ). Upon arrival and until 14 months of age, each mouse received $84 \mathrm{kcal}$ per week, approximately $10 \%$ lower than ad lib intake. Starting at 14 months of age, mice were randomly divided into one of three groups: a control group receiving the same diet; a calorie restricted (CR) group receiving $63 \mathrm{kcal}$ per week; or a resveratrol-treated (RESV) group receiving the same diet as the controls but supplemented with $50 \mathrm{mg}$ trans-resveratrol per kg diet (Sigma-Aldrich, St. Louis) - this translates to a dose of $4.9 \mathrm{mg}$ resvera- trol day ${ }^{-1} \mathrm{~kg}^{-1}$ body weight assuming a $35 \mathrm{~g}$ mouse. Details on feeding protocols for control and CR diets have been published previously [27]. At 30 months of age, tissues were collected from mice, flash-frozen in liquid nitrogen and stored at $-80^{\circ} \mathrm{C}$. All procedures were in compliance with the American Physiological Society's "Guiding principles for research involving animals and human beings" [28] and were approved by the Institutional Animal Care and Use Committee of the William S. Middleton Memorial Veterans Hospital (Madison, WI).

\subsection{Gene expression profiling}

RNA was extracted from 200-400 mg of epididymal white adipose tissue using Trizol (Life Technologies) and was processed following manufacturer's protocols (Affymetrix, CA). A single RNA sample from each mouse (control, $n=6$; CR, $n=8$; RESV, $n=5$ ) was hybridized to individual MouseGene 2.0 ST arrays (samples were not pooled). Signal intensity data were generated using RMA and probe sets were annotated with Entrez Gene identifiers (http://www.ncbi.nlm.nih.gov/gene). Data were processed as described previously [25]; briefly, probe sets that were annotated with multiple or no Entrez Gene identifiers were removed from the dataset; additionally, for the genes represented by more than one probe set, only that probe set with the highest signal intensity averaged across arrays was retained for analysis. This filtering procedure resulted in a total of 22,327 unique genes.

Four publicly-available, peer-reviewed gene expression datasets were obtained from the National Center for Biotechnology's Gene Expression Omnibus (http://www.ncbi.nlm.nih.gov/geo/): GSE2952 [29], GSE25401 [30], GSE6718 [31] and GSE41168 [32]. These datasets were processed as described above.

\subsection{Data analysis}

Signal intensity data were $\log _{2}$ adjusted for normality; a gene was considered to be differentially expressed when $p<0.01$ using a two-tailed $t$-test (CR vs. control or RESV vs. control). Pathway analysis was performed after assigning genes to pre-defined functional classes using the Gene Ontology hierarchy (http://www.geneontology.org). To identify pathways that were modulated by CR and/or RESV, we used 
Parametric Analysis of Gene Set Enrichment (PAGE; 33). This algorithm can detect changes in the regulation of pathways even when there are modest, yet consistent, changes in the expression of individual genes. We defined a statistically significant change in a pathway when $p<0.001$.

\section{Results and discussion}

\subsection{Does resveratrol mimic CR in mouse WAT?}

Gene expression profiling analysis revealed 2,513 and 364 genes that were differentially expressed in response to long-term CR or RESV, respectively. Although the number of genes modulated by RESV in WAT was lower than was observed previously in other mouse tissues [26], PAGE analysis revealed that a similar number of pathways were modulated by each treatment (570 and 376 pathways were significantly regulated by CR and RESV, respectively). As shown in Figs. 1a and 1b, for the subset of 165 genes and 267 pathways that were significantly changed by both CR and RESV, there was $100 \%$ mimicry of CR by RESV

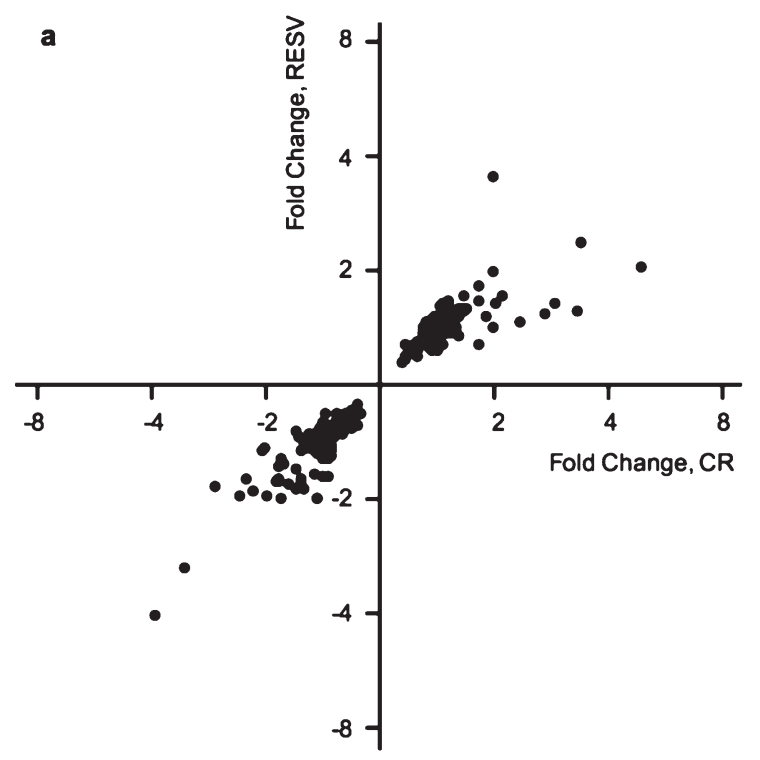

(i.e., if a gene or pathway was upregulated by $\mathrm{CR}$, it was also upregulated by RESV). This pattern mirrors what was observed in a previous study, where there was $>99 \%$ mimicry of CR by RESV at the gene expression level in brain, heart and skeletal muscle. Thus, the current results in mouse WAT add to the growing body of literature demonstrating that RESV mimics the effect of CR at the transcriptional level [22, 23].

\subsection{Identification of transcriptional targets of healthspan in WAT}

Because obesity and CR are strongly associated with age-associated disease, gene expression patterns that are modulated by energy intake represent promising targets for interventions designed to increase healthspan [16]. Moreover, obesity and CR-induced changes in WAT activity that are co-regulated in different species may hold even greater prospect as targets for interventions intended to slow the aging process. In an effort to identify such pathways, we analyzed gene expression datasets of obesity and CR from WAT in multiple species. Of the 5,160 pathways represented across all datasets, 22 pathways were significantly

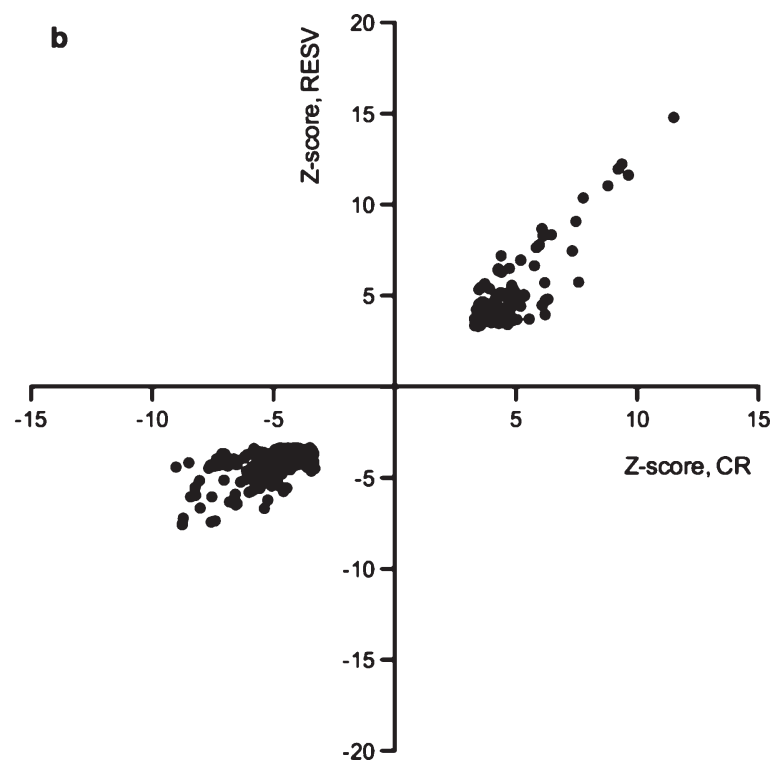

Fig. 1. Dietary consumption of resveratrol (RESV) from 14-30 months of age mimics the effect of a long-term calorie restricted (CR) diet in white adipose tissue (WAT) of mice for both individual genes and pathways. a. Each point represents an individual gene that was differentially expressed $(p<0.01)$ by both CR and RESV, values represent the fold change in gene expression relative to age-matched control mice. For the 165 genes changed in expression by both CR and RESV, gene expression changes were in the same direction for both treatments. b. Each dot represents a pathway (Gene Ontology term) that was differentially regulated $(p<0.001)$ by both CR and RESV; values are the $Z$-scores from pathway analysis (PAGE, see Materials and Methods) and represent the magnitude of the regulation of that pathway. For the 267 pathways regulated by CR and RESV, all pathways were regulated in the same direction by both treatments. 


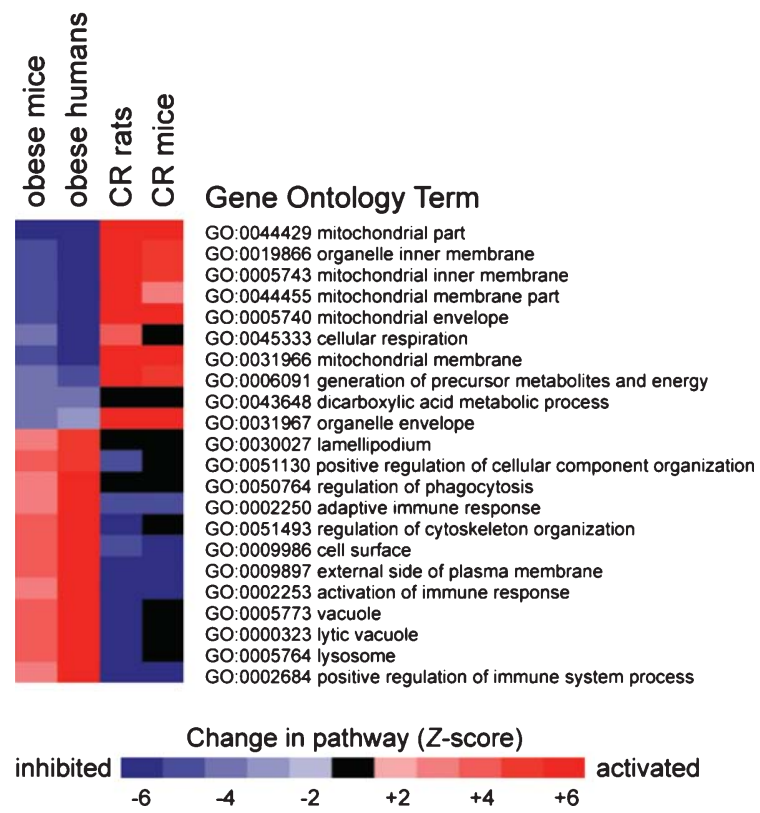

Fig. 2. Pathways regulated in WAT of both obese mice and humans tend to be regulated in the opposite direction by long-term CR in both mice and rats. Each column represents the results from pathway (PAGE) analysis of an independent dataset, each row represents a pathway (Gene Ontology term). The color represents the $Z$-score from PAGE, with red or blue indicating significantly $(p<0.001)$ increased or decreased activity, respectively (black fill indicates that a pathway was not statistically changed). Twenty-two pathways were modulated in both mouse and human obesity, and the effect of obesity was only opposed in both CR datasets for 13/22 pathways.

$(p<0.001)$ modulated by obesity in both mice [29] and humans [30]. As shown in Fig. 2, pathways related to mitochondrial structure and function (e.g., "mitochondrial part", "cellular respiration") were down-regulated with obesity and pathways related to the immune response and lysosomal function were up-regulated with obesity. This pattern tended to be reversed by CR, with CR significantly opposing the effect of obesity in both mice and rats for 13 pathways.

The contrasting modulation of mitochondrial and immune response pathways by obesity and CR for has been observed previously [34-36], however this is the first analysis to demonstrate that this pattern is similar across species. This shared pattern across taxa provides quantitative support for the hypothesis that mitochondrial metabolism and the immune/inflammatory response are critical regulators of healthspan [37-39]. We also observed an activation of pathways related to lysosomal function in WAT from obese mice and humans, although these pathways (e.g., "lysosome", regulation of phagocytosis") were not consistently downregulated by CR in both mice and rats. Increased activity of lysosomal pathways in obesity has been reported previously and may be associated with macrophage infiltration in obesity [40, 41]. Alternatively, the activation of lysosomal pathways in obesity may represent increased autophagic clearance of dysfunctional mitochondria [42] which is in agreement with our observation of decreased mitochondrial structure pathways observed in obesity. Although increased autophagy has been reported in the heart in response to CR [43], we observed a decrease in phagocytosis and lysosomal pathways in CR rats, but no change in mice. Further studies will be required to determine if autophagy is a critical mechanism linking diet and healthspan, or if this process is tissue-specific.

\subsection{Does resveratrol modulate healthspan targets in WAT?}

Our analyses revealed 13 pathways related to mitochondrial structure and the immune response that represent potential targets for interventions designed to increase healthspan. Our analysis of gene expression data from nonobese women with normal glucose tolerance [32] revealed that 12 weeks of resveratrol supplementation $\left(75 \mathrm{mg} \mathrm{day}^{-1}\right)$ significantly opposed 


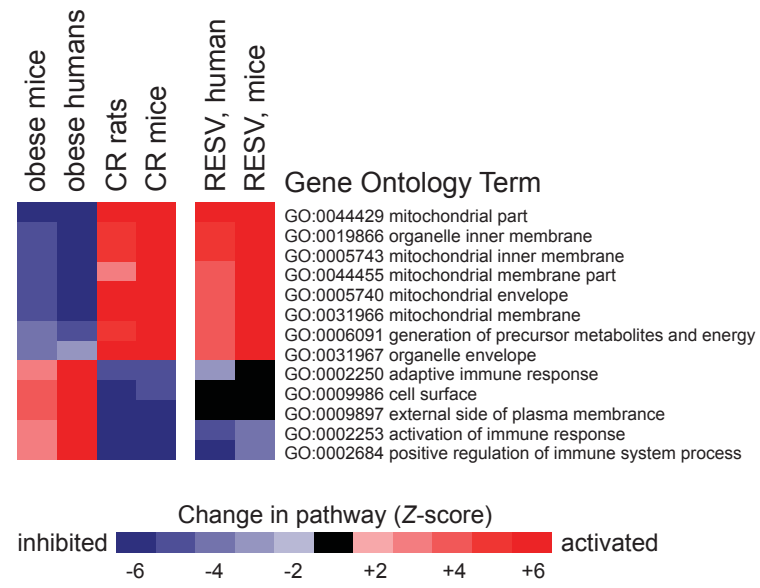

Fig. 3. Short-term RESV in metabolically healthy humans and long-term RESV in mice opposed the effect of obesity and partially mimicked the effect of $\mathrm{CR}$ for the 13 pathways that were modulated in all datasets shown in Fig. 2. Each column represents the results from pathway analysis of a gene expression dataset; the color represents the $Z$-score from PAGE with red and blue indicating significantly $(p<0.001)$ increased or decreased activity, respectively (black fill indicates that a pathway was not statistically changed).

the effect of obesity and mimicked the effect of CR for $11 / 13$ pathways; in the current long-term feeding study of mice, we found a similar effect of RESV for 10/13 pathways (Fig. 3). Interestingly, a re-analysis of gene expression data from in vastus lateralis muscle of obese males consuming $150 \mathrm{mg}$ resveratrol day ${ }^{-1}$ for 30 days revealed an upregulation of the same mitochondrial pathways and a downregulation of immune response pathways [44]. Other studies have shown that resveratrol consumption improves biomarkers of healthspan including decreased cataracts, preservation of motor function and bone mineral density [23], prevention of age-related cardiac dysfunction [26], improvements in mitochondrial function and insulin sensitivity and decreases in blood pressure and circulating inflammatory markers [45]. Taken together, these findings support the hypothesis that resveratrol has the potential to improve healthspan, possibly by mimicking CR at the gene expression level in WAT. Nonethless, Poulsen et al. [46] found no improvements in mitochondrial function or inflammation when resveratrol was administered to obese males at a dose 10-fold higher than the study by Timmers et al. [45]. It is therefore possible that the effects of resveratrol (and other CR mimetics) are dose-dependent.

In summary, our analysis of gene expression data in WAT revealed a set of 13 pathways that are differentially modulated under conditions of obesity and CR. These pathways are relevant to healthspan and may be useful for assessing the efficacy of CR mimetics, as the amount and activity of adipose tissue has been linked to diverse age-related diseases. In agreement with this hypothesis, we have shown that consumption of resveratrol by humans and mice mimics the effect of CR for 11 and 10 of these pathways, respectively. The genes, proteins and/or metabolic pathways identified in our analysis may be useful screening tools for identifying novel CR mimetics.

\section{References}

[1] McCay CM, Crowell MF. The effect of retarded growth upon the length of life span and upon the ultimate body size. Journal of Nutrition 1935;10(1):63-79.

[2] Weindruch R, Walford RL. The Retardation of Aging and Disease By Dietary Restriction. Springfield, Ill.: Charles C Thomas; 1988;436.

[3] Colman RJ, Anderson RM, Johnson SC, Kastman EK, Kosmatka KJ, Beasley TM, et al. Caloric restriction delays disease onset and mortality in rhesus monkeys. Science 2009; 325(5937):201-4.

[4] Mattison JA, Roth GS, Beasley TM, Tilmont EM, Handy AM, Herbert RL, et al. Impact of caloric restriction on health and survival in rhesus monkeys from the NIA study. Nature 2012;489(7415):318-21.

[5] Fontana L, Meyer TE, Klein S, Holloszy JO. Long-term calorie restriction is highly effective in reducing the risk for atherosclerosis in humans. Proc Natl Acad Sci U S A 2004;101(17): 6659-63.

[6] Pugh TD, Conklin MW, Evans TD, Polewski MA, Barbian HJ, Pass R, et al. A shift in energy metabolism anticipates the onset of sarcopenia in rhesus monkeys. Aging Cell 2013;12(4): 672-81. 
[7] Gresl TA, Colman RJ, Roecker EB, Havighurst TC, Huang Z, Allison DB, et al. Dietary restriction and glucose regulation in aging rhesus monkeys: A follow-up report at $8.5 \mathrm{yr}$. American Journal of Physiology Endocrinology and Metabolism 2001;281(4):E757-65.

[8] Falcao-Pires I, Castro-Chaves P, Miranda-Silva D, Lourenco AP, Leite-Moreira AF. Physiological, pathological and potential therapeutic roles of adipokines. Drug Discov Today. 2012;17(15-16):880-9.

[9] Bastard JP, Maachi M, Lagathu C, Kim MJ, Caron M and Vidal $\mathrm{H}$, et al. Recent advances in the relationship between obesity, inflammation, and insulin resistance. Eur Cytokine Netw 2006;17(1):4-12.

[10] Festa A, D’Agostino R, Jr., Williams K, Karter AJ, MayerDavis EJ, Tracy RP, et al. The relation of body fat mass and distribution to markers of chronic inflammation. Int J Obes Relat Metab Disord 2001;25(10):1407-15.

[11] Jagust W, Harvey D, Mungas D, Haan M. Central obesity and the aging brain. Arch Neurol 2005;62(10):1545-8.

[12] Jensen GL. Inflammation: Roles in aging and sarcopenia. JPEN J Parenter Enteral Nutr 2008;32(6):656-9.

[13] LeRoith D, Novosyadlyy R, Gallagher EJ, Lann D, Vijayakumar A, Yakar S. Obesity and type 2 diabetes are associated with an increased risk of developing cancer and a worse prognosis; epidemiological and mechanistic evidence. Exp Clin Endocrinol Diabetes. 2008;116(Suppl 1):S4-6.

[14] Barzilai N, Gabriely I. The role of fat depletion in the biological benefits of caloric restriction. J Nutr 2001;131(3):903S-6S

[15] Ingram DK, Zhu M, Mamczarz J, Zou S, Lane MA, Roth GS, et al. Calorie restriction mimetics: An emerging research field. Aging Cell 2006;5(2):97-108.

[16] Minor RK, Allard JS, Younts CM, Ward TM, de Cabo R. Dietary interventions to extend life span and health span based on calorie restriction. J Gerontol A Biol Sci Med Sci 2010;65(7):695-703.

[17] Renaud S, de Lorgeril M. Wine, alcohol, platelets, and the French paradox for coronary heart disease. Lancet 1992;339(8808):1523-6.

[18] Jang M, Cai L, Udeani GO, Slowing KV, Thomas CF, Beecher $\mathrm{CW}$, et al. Cancer chemopreventive activity of resveratrol, a natural product derived from grapes. Science 1997;275(5297): 218-20.

[19] Howitz KT, Bitterman KJ, Cohen HY, Lamming DW, Lavu S, Wood JG, et al. Small molecule activators of sirtuins extend Saccharomyces cerevisiae lifespan. Nature 2003;425(6954): 191-6.

[20] Wood JG, Rogina B, Lavu S, Howitz K, Helfand SL, Tatar $\mathrm{M}$, et al. Sirtuin activators mimic caloric restriction and delay ageing in metazoans. Nature 2004;430(7000):686-9.

[21] Valenzano DR, Terzibasi E, Genade T, Cattaneo A, Domenici L, Cellerino A. Resveratrol prolongs lifespan and retards the onset of age-related markers in a short-lived vertebrate. Curr Biol 2006;16(3):296-300.

[22] Baur JA, Pearson KJ, Price NL, Jamieson HA, Lerin C, Kalra A, et al. Resveratrol improves health and survival of mice on a high-calorie diet. Nature 2006;444(7117):337-42.

[23] Pearson KJ, Baur JA, Lewis KN, Peshkin L, Price NL, Labinskyy N, et al. Resveratrol delays age-related deterioration and mimics transcriptional aspects of dietary restriction without extending life span. Cell Metab 2008;8(2):157-68.
[24] Strong R, Miller RA, Astle CM, Baur JA, de Cabo R, Fernandez $\mathrm{E}$, et al. Evaluation of resveratrol, green tea extract, curcumin, oxaloacetic acid, and medium-chain triglyceride oil on life span of genetically heterogeneous mice. J Gerontol A Biol Sci Med Sci 2012;68(1):6-16

[25] Barger JL, Kayo T, Pugh TD, Prolla TA, Weindruch R. Shortterm consumption of a resveratrol-containing nutraceutical mixture mimics gene expression of long-term caloric restriction in mouse heart. Exp Gerontol 2008;43(9):859-66.

[26] Barger JL, Kayo T, Vann JM, Arias EB, Wang J, Hacker TA, et al. A low dose of dietary resveratrol partially mimics caloric restriction and retards aging parameters in mice. PLOS ONE 2008;3(6):e2264.

[27] Pugh TD, Klopp RG, Weindruch R. Controlling caloric consumption: Protocols for rodents and rhesus monkeys. Neurobiol Aging 1999;20(2):157-65.

[28] American Physiological Society. Guiding principles for research involving animals and human beings. Am J Physiol Regul Integr Comp Physiol. 2002;283(2):R281-3.

[29] Nadler ST, Stoehr JP, Schueler KL, Tanimoto G, Yandell BS, Attie AD. The expression of adipogenic genes is decreased in obesity and diabetes mellitus. Proc Natl Acad Sci U S A 2000; 97(21):11371-6.

[30] Arner E, Mejhert N, Kulyte A, Balwierz PJ, Pachkov M, Cormont $\mathrm{M}$, et al. Adipose tissue microRNAs as regulators of CCL2 production in human obesity. Diabetes 2012;61(8): 1986-93.

[31] Linford NJ, Beyer RP, Gollahon K, Krajcik RA, Malloy VL, Demas V, et al. Transcriptional response to aging and caloric restriction in heart and adipose tissue. Aging Cell 2007;6(5): 673-88.

[32] Yoshino J, Conte C, Fontana L, Mittendorfer B, Imai S, Schechtman KB, et al. Resveratrol supplementation does not improve metabolic function in nonobese women with normal glucose tolerance. Cell Metab 2012;16(5):658-64.

[33] Kim SY, Volsky DJ. PAGE: Parametric analysis of gene set enrichment. BMC Bioinformatics 2005;6:144.

[34] Higami Y, Barger JL, Page GP, Allison DB, Smith SR, Prolla TA, et al. Energy restriction lowers the expression of genes linked to inflammation, the cytoskeleton, the extracellular matrix, and angiogenesis in mouse adipose tissue. J Nutr 2006;136(2):343-52.

[35] Higami Y, Pugh TD, Page GP, Allison DB, Prolla TA, Weindruch R. Adipose tissue energy metabolism: Altered gene expression profile of mice subjected to long-term caloric restriction. FASEB J 2004;18(2):415-7.

[36] Hotamisligil GS. Inflammation and metabolic disorders. Nature 2006;444(7121):860-7.

[37] Anderson RM, Weindruch R. Metabolic reprogramming, caloric restriction and aging. Trends in endocrinology and metabolism: TEM 2010;21(3):134-41.

[38] Schwanstecher C, Schwanstecher M. Targeting type 2 diabetes. Handbook of Experimental Pharmacology 2011;(203):1-33.

[39] Park S, Park NY, Valacchi G, Lim Y. Calorie restriction with a high-fat diet effectively attenuated inflammatory response and oxidative stress-related markers in obese tissues of the high diet fed rats. Mediators of Inflammation 2012;2012: 984643.

[40] Gornicka A, Fettig J, Eguchi A, Berk MP, Thapaliya S, Dixon $\mathrm{LJ}$, et al. Adipocyte hypertrophy is associated with lysosomal 
permeability both in vivo and in vitro: Role in adipose tissue inflammation. American Journal of Physiology Endocrinology and Metabolism 2012;303(5):E597-606.

[41] Grant RW, Vester Boler BM, Ridge TK, Graves TK, Swanson KS. Subcutaneous and gonadal adipose tissue transcriptome differences in lean and obese female dogs. Animal Genetics 2013.

[42] Salminen A, Kaarniranta K, Kauppinen A. Inflammaging: Disturbed interplay between autophagy and inflammasomes. Aging 2012;4(3):166-75.

[43] Wohlgemuth SE, Julian D, Akin DE, Fried J, Toscano K, Leeuwenburgh C, et al. Autophagy in the heart and liver during normal aging and calorie restriction. Rejuvenation Research 2007;10(3):281-92.
[44] Barger JL. An adipocentric perspective of resveratrol as a calorie restriction mimetic. Annals of the New York Academy of Sciences 2013;1290(1):122-9.

[45] Timmers S, Konings E, Bilet L, Houtkooper RH, van de Weijer T, Goossens GH, et al. Calorie restriction-like effects of 30 days of resveratrol supplementation on energy metabolism and metabolic profile in obese humans. Cell Metab 2011;14(5):612-22.

[46] Poulsen MM, Vestergaard PF, Clasen BF, Radko Y, Christensen LP, Stodkilde-Jorgensen H, et al. High-Dose Resveratrol Supplementation in Obese Men: An Investigator-Initiated, Randomized, Placebo-Controlled Clinical Trial of Substrate Metabolism, Insulin Sensitivity, and Body Composition. Diabetes 2012 . 